\title{
Processo de trabalho na atenção básica à saúde: a utilização do atendimento individual específico e articulação intersetorial por terapeutas ocupacionais e psicólogos
}

\section{Work process in primary health care: the use of specific individual care and intersectoral articulation by occupational therapists and psychologists}

\author{
Derivan Brito da Silva ${ }^{1}$, Priscila Moreira ${ }^{1}$, Lilian Cristina dos Santos ${ }^{1}$, Maria José \\ Gugelmin de Camargo ${ }^{1}$, Denny Rafael Perusso dos Reis ${ }^{1}$
}

http://dx.doi.org/10.11606/issn.2238-6149.v30i2p86-93

Silva DB, Moreira P, Santos LC, Camargo MJG, Reis DRP. Processo de trabalho na atenção básica à saúde: a utilização do atendimento individual específico e articulação intersetorial por terapeutas ocupacionais e psicólogos. Rev Ter Ocup Univ São Paulo. 2020 maio-ago.;30(2):86-93.

RESUMO: A reorientação do modelo assistencial, no contexto da Atenção Básica à Saúde (ABS), com a implantação das equipes de Saúde da Família (SF) e do Núcleo Ampliado de Saúde da Família e Atenção Básica (NASF-AB) possibilitou aproximação entre os profissionais e comunidade. Esta aproximação facilita o acesso dos usuários e o manejo dos objetos de ação pelos profissionais da ABS. O objetivo deste trabalho foi o de identificar as ferramentas utilizadas por terapeutas ocupacionais e psicólogos da equipe do NASF-AB no processo de trabalho, junto às equipes de $\mathrm{SF}$, para a assistência a população. Para isto, foi realizada pesquisa bibliográfica, documental e de campo. Constata-se que para intervir sobre as demandas presentes no território de abrangência das Unidades Básicas de Saúde (UBS), os profissionais do NASF-AB enquanto agentes, utilizam como meios de produção ferramentas tecnológicas e de cuidado, como o atendimento individual específico, com os objetivos de prevenir agravos e promover saúde física e psíquica a população.

DESCRITORES: Sistema Único de Saúde; Atenção primária à saúde; Terapia ocupacional.
Silva DB, Moreira P, Santos LC, Camargo MJG, Reis DRP. Work process in primary health care: the use of specific individual care and intersectoral articulation by occupational therapists and psychologists. Rev Ter Ocup Univ São Paulo. 2020 MayAug.;30(2):86-93.

ABSTRACT: The reorientation of the care model, in the context of Basic Care in Health (BCH), with deployment of Family Health (FH) teams and the Expended Nucleus of Family Health and Basic Care (ENFH-BC) allowed the approach between professionals and community. This approach made the access of users and the management of objects of action by the $\mathrm{BCH}$ professionals easier. The objective of this paper was to identify the tools used by occupational therapists and psychologists of the ENFH-BC team in the work process, together with the FH team, to assist the population. For this, the bibliographical, documentary and field research was opened. It appears that to intervene on the demands presente in the teritory covered by the Basic Health Units, the professionals of ENFH-BC as agents, use as a means of production technological tools and care, such as specific individual care, with the objectives of preventing the aggravations and promoting physical and mental health to the population.

KEYWORDS: Unified health system; Primary health care; Occupational therapy.

Este trabalho é parte integrante ao Projeto de Pesquisa: Formação, Exercício e Regulamentação Profissional em Terapia Ocupacional: Construindo Caminhos no Contexto da Atenção Básica À Saúde. Trabalho de Conclusão de Residência (TCR).

1. Universidade Federal do Paraná. ORCID: Silva DB - https://orcid.org/0000-0002-0971-7441; Moreira P - https://orcid.org/0000-0002-4453363X; Santos LC - https://orcid.org/0000-0001-8092-7831; Camargo MJG - https://orcid.org/0000-0003-1698-3163; Reis DRP - https://orcid. org/0000-0002-6936-721X.Email: derivan_to@hotmail.com; priscilamoreira.to@gmail.com; liliancris@gmail.com; mariajosegugelmin@ hotmail.com; mariajosegugelmin@hotmail.com; reisdenny@yahoo.com.

Endereço para correspondência: Av. Prefeito Lothário Meissner, 632. Jardim Botânico. Curitiba, PR. CEP. 80.210-170. 


\section{INTRODUÇÃO}

Os Núcleos Ampliados de Saúde da Família e Atenção Básica (NASF-AB) são equipes multiprofissionais que tem por finalidade apoiar as equipes de Saúde da Família (SF) e Atenção Básica $(\mathrm{AB})^{1}$. Terapeutas ocupacionais e psicólogos são profissionais que podem compor os NASFAB. Entre as ferramentas de trabalho utilizadas por estes profissionais estão: o Atendimento Individual Específico e a Articulação Intersetorial. Assim, por meio dos NASF-AB, terapeutas ocupacionais e psicólogos se inserem no Processo de Trabalho da ABS. Faria et al. $^{2}$ aponta a ideia de que o processo de trabalho no contexto da Atenção Básica à Saúde (ABS) é formado por quatro elementos interdependentes e que se relacionam para produzir cuidado a população, a saber: (1) Meios de produção, (2) Objetivos e Finalidades (3) Objeto de ação e (4) Agentes.

Os meios de produção são constituídos por: estrutura física das unidades, equipamentos e suplementos técnicos; conhecimentos e habilidades dos profissionais e os meios relacionados à interação dos profissionais entre si e destes com os usuários-comunidade ${ }^{2}$. Para desenvolver suas ações os profissionais do NASF-AB utilizam como meios de produção as ferramentas tecnológicas de: apoio matricial, clínica ampliada, Projeto Terapêutico Singular (PTS) e o Projeto Saúde no Território (PST). Em paralelo, as ferramentas de cuidado são colocadas pela literatura, como meios para operacionalizar o apoio matricial, sendo estas: Atendimento individual específico, atendimento compartilhado, atendimentos grupais, genograma, ecomapa e visita domiciliar compartilhada ${ }^{3,4}$.

No caso do NASF-AB, para ofertar o apoio matricial os profissionais partem de duas dimensões, a saber: (1) clínicoassistencial e (2) técnico-pedagógica ${ }^{1,4}$. A dimensão clínicoassistencial refere-se às ações de cuidado direcionadas para os usuários como, por exemplo, o atendimento individual específico. Já a dimensão técnico-pedagógica refere-se a ações educativas realizadas para e com os profissionais das equipes de SF como, por exemplo, as discussões de casos.

Em relação aos objetivos e finalidades, estes se referem às expectativas e respostas esperadas pelos indivíduos e por grupos constituídos. Prevenir doenças e agravos e promover a saúde são objetivos centrais do processo de trabalho na $\mathrm{ABS}^{2}$. Os profissionais do NASFAB utilizam ferramentas tecnológicas e de cuidado visando apoiar às equipes de SF para que estes profissionais possam executar ações preventivas e de assistência à população em suas necessidades de saúde.

Os profissionais do contexto da ABS atuam sobre o processo saúde-doença, intervindo sobre um objeto de ação de três dimensões: biológica, psíquica e social. Assim, as ações das equipes de SF e do NASF-AB precisam ser direcionadas a coletivos, com atuação sobre riscos e vulnerabilidades da população, considerando não somente aspectos biológicos, mas sociais e subjetivos de indivíduos e coletividades de maneira direta e/ou indireta 4 .

Em relação aos agentes, temos: o sistema público de saúde, equipes de saúde e a população local ${ }^{2}$. As equipes de SF e do NASF-AB foram implementadas pelo Ministério da Saúde (MS) com o objetivo de reorientar o modelo assistencial em saúde, em especial na ABS. A implantação destas equipes representou importante avanço para a produção de saúde baseada na perspectiva da determinação social do processo saúde-doença ${ }^{5}$.

O processo de trabalho na ABS é composto por estes quatro elementos e norteado por diretrizes, sendo uma destas a Articulação Intersetorial. Os profissionais do NASF-AB desempenham papel importante para articular com outros pontos da rede assistencial a fim de apoiar as equipes de SF para que estas consigam atender a população em todas as suas necessidades.

O objetivo deste estudo foi o de identificar as ferramentas utilizadas por terapeutas ocupacionais e psicólogos das equipes do NASF-AB, de um município da Região Metropolitana de Curitiba/Paraná, para assistência clínica e para apoio das equipes de saúde família.

A motivação para exploração deste tema decorreu da vivência prática e discussão teórica dos autores enquanto agentes (residentes, preceptores e tutor) do Programa de Residência Multiprofissional em Saúde da Família da Universidade Federal do Paraná.

Ressalta-se que este estudo é parte integrante do projeto de pesquisa intitulado: "Formação, Exercício e Regulamentação Profissional em Terapia Ocupacional: Construindo caminhos no contexto da Atenção Básica à Saúde”, aprovado pelo Comitê de Ética e Pesquisa da Universidade Federal do Paraná (UFPR) sob o número CAAE 95581918.4.0000.0102.

\section{MÉTODOS}

Trata-se de um estudo qualitativo de caráter exploratório e descritivo ${ }^{6,7}$ que utilizou três procedimentos de pesquisa: Bibliográfica, documental ${ }^{8}$ e de campo ${ }^{10}$. Em relação à pesquisa bibliográfica foram consultados livros e materiais online. Na pesquisa documental foram consultadas normativas relacionadas ao contexto da ABS e ao objeto de pesquisa. Para a pesquisa de campo foram realizadas entrevistas e observação participante com o objetivo de coletar e produzir dados empíricos ${ }^{9,10,11}$. 
Para as entrevistas foi utilizado roteiro semiestruturado ${ }^{10,11}$ com questões relacionadas a processos e ferramentas de trabalho, dificuldades e facilidades de atuação no contexto da ABS, entre outras.

\section{Participantes}

Foram entrevistados 03 psicólogos, sendo identificados pelas siglas: P1; P2 e P3 e 03 terapeutas ocupacionais identificados pelas siglas: T1; T2 e T3, de equipes do NASF$\mathrm{AB}$ de um município da Região Metropolitana de Curitiba/ Paraná. O tempo de atuação dos profissionais nas equipes do NASF-AB variou de 04 meses a 02 anos.

Tabela 1 - Perfil dos profissionais entrevistados

\begin{tabular}{lcccc}
\hline $\begin{array}{l}\text { Categoria } \\
\text { Profissional }\end{array}$ & Sexo & $\begin{array}{c}\text { Tempo de } \\
\text { formação }\end{array}$ & $\begin{array}{c}\text { Tempo de atuação } \\
\text { no NASF }\end{array}$ & $\begin{array}{c}\text { Sigla } \\
\text { utilizada }\end{array}$ \\
\hline Psicologia & $\mathrm{M}$ & 7 anos & 04 meses & P1 \\
Psicologia & M & 5 anos & 01 ano & P2 \\
$\begin{array}{l}\text { Psicologia } \\
\text { Terapia }\end{array}$ & F & $\begin{array}{c}10 \text { anos } \\
1 \text { ano e }\end{array}$ & $\begin{array}{c}05 \text { meses } \\
1 \text { ano e }\end{array}$ & P3 \\
$\begin{array}{l}\text { Ocupacional } \\
\text { Terapia }\end{array}$ & F & 6 meses & 6 meses & T1 \\
$\begin{array}{l}\text { Ocupacional } \\
\text { Terapia }\end{array}$ & F & 07 meses & 07 meses & T2 \\
Ocupacional & F & 09 anos & 02 anos & T3 \\
\hline
\end{tabular}

As entrevistas foram realizadas as categorias profissionais consideradas referência para a saúde mental ao compor a equipe NASF-AB, considerando a aproximação do objeto de ação destes profissionais com o das terapeutas ocupacionais inseridas nas equipes do NASF-AB do município.

\section{Campo de estudo}

Foram entrevistados terapeutas ocupacionais e psicólogos de três equipes do NASF-AB, de um município da Região Metropolitana de Curitiba, de modalidade do tipo 01 , sendo estes compostos por diferentes categorias profissionais, conforme Política Nacional da $\mathrm{ABS}^{1}$. O estudo foi composto pelas seguintes etapas: (1) exploração do tema, (2) coleta de dados (3) análise dos dados e (4) discussão. A exploração do tema foi realizada no período de fevereiro de 2018 a fevereiro de 2019. A coleta de dados, composta por diário de campo e entrevistas, foi realizada no período de setembro a novembro de 2018. A análise dos dados ${ }^{10,11}$ foi realizada no período de novembro a janeiro de 2019 e posteriormente foi realizada a discussão dos achados empíricos e teóricos.

\section{Procedimentos}

\section{Coleta de dados}

Para as entrevistas foi utilizado um roteiro semiestruturado com questões relativas ao perfil dos profissionais, experiências prévias, processo e ferramentas de trabalho, dificuldades encontradas para atuação no contexto do NASF-AB, assim como as potencialidades existentes neste contexto de prática. Os profissionais foram convidados para participar da entrevista por meio de contato pessoal e telefônico. As entrevistas foram realizadas no local de prática do profissional.

Em relação à observação participante foi elaborado o Diário de Campo ${ }^{7,8,11}$ no qual foram registradas observações referentes à: (1) dinâmica das unidades básicas de saúde onde atua a pesquisadora e participantes da pesquisa; (2) reuniões de saúde mental e da rede de proteção; (3) as práticas assistenciais e gerenciais em torno da temática da saúde mental.

\section{Análise dos dados}

A análise de dados foi realizada por meio de análise operativa composta por três etapas: ordenação dos dados, classificação dos dados e análise dos dados ${ }^{10,11}$.

A etapa da ordenação consistiu inicialmente na organização dos áudios das entrevistas e dos registros do diário de campo.

$\mathrm{Na}$ etapa de classificação e análise dos dados foi realizada leitura exaustiva dos achados em campo sendo estes agrupados em torno de categorias centrais, a fim de elaborar uma lógica unificada ${ }^{10,11}$. As categorias centrais estavam relacionadas em parte com as ferramentas de trabalho do NASF-AB e diretrizes da Atenção Básica à Saúde ${ }^{1,3,4}$. Outra parte daquelas categorias necessitava de um aporte teórico em prol da lógica unificada. Assim foi optado por operacionalizar a classificação em dois momentos.

Primeiramente, tomou-se os elementos do processo de trabalho da ABS, enquanto categorias analíticas, a saber: (1) objetivos e finalidades; (2) objeto; (3) meios de produção; e (4) agentes ${ }^{2}$. A partir destas categorias analíticas buscouse compreender o objeto de investigação - ferramentas assistenciais e tecnológicas utilizadas por terapeutas ocupacionais e psicólogos do NASF-AB - na perspectiva dos participantes.

Em seguida, o terceiro elemento - meios de produção - foi selecionado para seguir com a análise e discussão. Nesta etapa, foram nomeadas e reagrupadas as ferramentas de trabalho do NASF-AB e as diretrizes da Atenção Básica à Saúde, publicadas pelo Ministério 
da Saúde ${ }^{1,4}$. Do conjunto das ferramentas e diretrizes identificadas, foram selecionadas: (1) ferramentas: atendimento individual específico, atendimentos grupais e apoio matricial; (2) diretriz: articulação intersetorial. Esta seleção buscou aproximar tais categorias analíticas do objeto de investigação - ferramentas assistenciais e tecnológicas utilizadas por terapeutas ocupacionais e psicólogos do NASF$\mathrm{AB}-\mathrm{em}$ acordo com as perspectivas dos participantes da pesquisa, das políticas públicas envolvidas e das ideias em torno do processo de trabalho na $\mathrm{ABS}^{2}$.

Estes dois momentos permitiram a elaboração da análise final, de forma a elaborar uma lógica unificada, facilitar a exposição e explicação do nosso objeto de investigação e alcance do objetivo proposto. Porém, para este estudo, foram selecionadas apenas três categorias: Meios de produção, atendimento individual específico e articulação intersetorial, para que estas possam ser abordadas com maior profundidade.

\section{RESULTADOS}

Os resultados apresentados, a seguir, sintetizam os achados de campo referentes às categorias: Meios de Produção, Atendimento Individual Específico e Articulação Intersetorial. Os participantes da pesquisa discorreram nas entrevistas sobre estratégias e recursos que utilizam em sua prática assistencial e ao prestar apoio para as equipes de saúde, comentando sobre os desafios e potencialidades que encontram no campo da ABS.

\section{Processo de trabalho na atenção básica à saúde: meios de produção}

Em relação aos meios de produção utilizados no contexto da ABS, os profissionais entrevistados referem recursos e estratégias que podem auxiliar tanto a população atendida quanto à equipe de saúde da família, e citam alguns exemplos de situações que são encaminhadas e casos em que são solicitados para apoiar a fim de elucidar como se dá a dinâmica do uso destes meios de produção no processo de trabalho da ABS.

Um dos profissionais entrevistados refere exemplos de meios utilizados para manejar um caso complexo no contexto da ABS:

"Foi feito um processo de atendimento individual, visita domiciliar, sensibilização e psicoeducação da equipe em entender que aquele comportamento [crise] foi pontual [...] O paciente frequenta a unidade até hoje por outras questões e está totalmente estável" $P 2$.
Também são citados exemplos de recursos terapêuticos específicos utilizados como meios de produção. A terapeuta ocupacional entrevistada exemplifica um atendimento individual prestado à vítima de violência intrafamiliar:

"Atividades como recurso para que a mulher consiga se expressar, o que é muito difícil para ela [...] Utilizando atividades expressivas como meio e também planejamento de vida" T1.

Quando os entrevistados são questionados de como realizam a escolha por qual meio utilizar em determinada situação, um dos profissionais entrevistados relata que:

"Sempre tento olhar de uma forma mais transversal, a participação nos grupos. Se eu percebo uma dificuldade do paciente funcionar em grupo já vejo a possibilidade de encaixar ele na agenda para atendimentos individuais" $P 3$.

Uma das entrevistadas refere a importância de refletir sobre meios que possam ser utilizados no contexto da ABS, além do atendimento individual, como podemos identificar na fala a seguir:

"Temos uma demanda de atendimento gigantesca, principalmente nesta região, porém se a gente não parar um pouco e planejar estratégias de atuação que produzam efeitos a longo prazo e que a gente consiga diminuir essa demanda de atendimento individual, diante de um trabalho de prevenção, não teremos efetividade nenhuma" $P 3$.

A mesma profissional acrescenta como facilitador do processo de trabalho na ABS a possibilidade de utilizar vários meios para a produção de cuidado:

"Temos a facilidade de estar em vários contextos [...] posso identificar uma situação e ao mesmo tempo fazer um atendimento compartilhado com a educadora fisica [...] posso estar em um atendimento individual, em uma consulta compartilhada, em uma ação na unidade, como posso participar de um grupo [...] há uma gama de recursos para poder tratar, acompanhar aquele usuário, isso é muito bom, muito positivo" $P 3$.

Os profissionais entrevistados relatam os meios de produção utilizados enfatizando o atendimento individual específico e ressaltam também a importância de articular com a rede para que o cuidado prestado seja efetivo:

"O que não foge à regra, é a articulação de rede, a gente sempre aciona os serviços, quando isso acontece [caso de violência intrafamiliar], porque é algo que foge da nossa capacidade, então a gente tem que acionar e o NASF tem esse papel articulador" T3. 
Serão apresentados, a seguir, os resultados encontrados referentes ao uso do atendimento individual específico pelos profissionais do NASF-AB, assim como a diretriz da articulação intersetorial, de modo a expor as dificuldades encontradas para operacionalização destes meios no contexto da ABS.

\section{Atendimento individual específico como ferramenta de cuidado da equipe NASF-AB}

Os profissionais entrevistados ao comentarem sobre a ferramenta do atendimento individual específico colocam que há grande demanda para que seja realizado este tipo de intervenção na $\mathrm{ABS}$ por profissionais do NASF-AB, sendo assim utilizam estratégias para o uso desta, assim como encontram algumas dificuldades para definir os critérios e objetivos desta modalidade de atendimento juntamente com as equipes de saúde família.

Uma das profissionais refere que existem espaços, como as discussões de casos, que poderiam servir como meio para que os objetivos destes atendimentos fossem discutidos, assim como para que as situações fossem compartilhadas entre as equipes, porém nem todos os profissionais se disponibilizam para isto:

"É discutido sobre a vida ou a situação do paciente e é como se você tivesse que dar conta de uma situação onde você precisa de todos os demais profissionais envolvidos para poder fazer as pontuações necessárias, para poder gerar certa mudança dentro do contexto social e familiar dessa pessoa para que ela possa ter melhora" P3.

Para os participantes, apesar de não ser o foco de intervenção e principal função dos profissionais da equipe do NASF-AB, a maior demanda que se apresenta pela equipe de SF são casos para atendimento individual:

"O que eu tenho usado como recurso é o atendimento individual [...] mesmo sabendo que não caberia dentro das unidades, porque eu estou fazendo muito mais ambulatório do que necessariamente minha função enquanto psicólogo" $P 3$.

Para enfrentar a barreira que se coloca para o uso da ferramenta do atendimento individual, considerando que este não deve ser o foco de trabalho da equipe do NASF$\mathrm{AB}$, os participantes colocam que estes atendimentos são realizados de maneira pontual, sendo utilizadas abordagens mais rápidas o que denominam de intervenção breve:

"Então a maioria dos pacientes que chegam aqui que eu percebo que consigo manejar na unidade fica aqui, fazendo uma média de 4, 5, 6 atendimentos individuais e depois começa atendimentos de apoio quinzenais [...] mas, sempre com um foco muito especifico sempre voltado para o que a gente chama de intervenção breve” P2.

Um dos participantes coloca sua opinião em relação ao discurso comum que existe sobre o uso da ferramenta do atendimento individual específico pelas equipes do NASF-AB:

"Claro que nosso enfoque é o apoio matricial da equipe, atendimentos em grupo, mas em nenhum momento eu vejo a literatura negando esse apoio de atendimento individualizado [...] não creio que isso foge do meu trabalho, acho muito complicado também o psicólogo do NASF fugir disso" $P 2$.

\section{A diretriz da articulação intersetorial para o cuidado de vítimas de violência intrafamiliar}

Em relação a articulação intersetorial, os profissionais relatam que utilizam esta diretriz principalmente em situações que são denominadas complexas e que exigem conhecimentos específicos de outras áreas para o manejo, como por exemplo, casos de violência intrafamiliar:

"A ideia não é lidar com a violência dentro da saúde, a assistência tem mais manejo para isso" P1.

Uma das terapeutas ocupacionais entrevistadas relata que enfrenta barreiras para colocar em prática esta ferramenta, pois nem sempre os demais equipamentos estão dispostos a compartilhar o cuidado:

“... a nossa rede de cuidado é bem frágil [...] por isso é muito dificil abordar uma pessoa, vítima de violência, que continua morando na mesma casa com o agressor [...] e que não tem suporte fora a gente [equipes de saúde da ABS] ” T1.

Outra terapeuta ocupacional entrevistada coloca que sente resistência das equipes de $\mathrm{SF}$ e dos demais profissionais do NASF-AB em utilizar esta ferramenta e que esta resistência também a desestimula em tentar trabalhar de forma intersetorial:

"Muitas vezes já ouvi: "tal serviço não funciona da forma adequada”, então já cria uma resistência sem eu ter tido contato prévio com esse equipamento. Acho que isso já me desestimula a pensar esse cuidado de forma intersetorial com outros equipamentos" T2.

A mesma participante refere que uma das dificuldades para pensar o trabalho intersetorial é o desconhecimento da função dos serviços pelos profissionais: 
"Participei de uma reunião de rede na semana passada [...] já senti certa dificuldade em pensar em rede [...] acho que se constitui a rede, mas ninguém ao certo sabe seu papel, há uma dificuldade grande para trabalhar em rede” $T 2$.

Uma das estratégias relatadas para facilitar o uso desta ferramenta seria desenvolver ações de saúde nos serviços de outras redes, como da assistência social, como forma de aproximação com os profissionais que compõe as equipes deste local:

"A gente faz bastante ação na escola, ação no abrigo que tem aqui, ação no CRAS. Então tentamos articular com os serviços, até foi uma estratégia minha de incentivar isso para a gente se aproximar dos serviços, porque eu senti dificuldades nessa articulação” T3.

De acordo com os relatos identifica-se que, apesar das barreiras encontradas para realizar a articulação intersetorial, os profissionais já buscam meios e estratégias para se aproximar de outros serviços a fim de que esta aproximação facilite o uso desta ferramenta nas situações necessárias.

\section{DISCUSSÃO}

Os profissionais do NASF-AB utilizam como meios de produção ferramentas assistenciais e tecnológicas que também são utilizadas pelos profissionais das equipes de SF apoiadas, assim como por equipes de outros serviços da rede assistencial. Pouca ênfase é dada a ferramentas específicas que podem ser utilizadas pelas equipes do NASF-AB ao apoiar as equipes de SF, como por exemplo: PTS, PST, genograma e ecomapa.

Em relação ao atendimento individual específico a literatura traz a importância de se discutir com as equipes de SF os objetivos do atendimento, a ser realizado por profissional da equipe do NASF-AB, que dependem das necessidades dos usuários e/ou famílias, existindo negociação e corresponsabilização por parte de todos os envolvidos ${ }^{4}$.

O uso da ferramenta do atendimento individual é enfatizado e há concordância sobre a cautela em sua utilização, pois não seria o foco de trabalho dos profissionais da equipe do NASF-AB. Porém são encontradas dificuldades em pensar em outras ferramentas, pois as demandas que são encaminhadas pelas equipes de SF, na maioria das situações, são casos que necessitam de atenção individualizada. Diante disto utilizam como estratégia a intervenção breve para que a ferramenta do atendimento individual possa alcançar maior número de usuários e para que sejam atendidos em suas necessidades individuais.
A questão do atendimento individual específico é complexa. Observa-se que há confusão entre esta estratégia e a clínica especializada baseada na lógica individualizadora psicobiologicista. Acredita-se que é possível utilizar a atenção individual para além desta lógica. Entretanto, esta confusão leva, por vezes, gestores a advertirem profissionais do NASF-AB acerca do atendimento individual, e também é comum profissionais do NASF-AB se indagarem ou indagar colegas durante o processo de trabalho se devem ou não, ou em que circunstâncias, podem realizar um atendimento individual específico.

Entende-se que o atendimento individual específico deve ser utilizado em acordo com as demandas individuais dos usuários que buscam atendimento, de forma articulada ao objeto do campo profissional daquele que presta a assistência. Talvez seja necessário compreender que o problema não é o uso da ferramenta, mas o que ela demanda para sua utilização, como por exemplo: As expertises dos profissionais que compõem a equipe NASF$\mathrm{AB}$, o repasse financeiro por procedimento - que privilegia as atividades coletivas -, a estrutura física das UBS e o deslocamento dos profissionais para o domicílio do usuário. Estes exemplos se concretizam na diversidade de problemáticas que constituem o perfil epidemiológico de um território de abrangência de uma Unidade Básica de Saúde.

Os profissionais entrevistados também referem dificuldades para operacionalizar a articulação intersetorial e consideram esta diretriz primordial ao tratar da questão da violência intrafamiliar, visto que esta possui elementos que são objetos de ação de outros serviços, como por exemplo, da assistência social. Sendo assim, esta diretriz será abordada, a seguir, sendo expostas as dificuldades encontradas para o uso desta pelos profissionais do NASF-AB.

Em relação a articulação intersetorial esta é definida como uma diretriz que pode ser utilizada para várias situações na ABS. Entendida como ações articuladas com o envolvimento de outros setores que tem por objetivo produzir saúde reconhecendo que esta se relaciona com a educação, segurança, cultura, habitação, assistência social, transporte, lazer e esporte. A equipe do NASFAB pode estabelecer parceria com a equipe de SF para o desenvolvimento desta articulação, pois os profissionais apoiadores relacionam-se com um número expressivo de casos em certos campos de atuação e por isso conhecem a rede assistencial ${ }^{4}$.

Assim como o atendimento individual específico, a articulação intersetorial envolve aspectos complexos: 
a característica do território; a composição da rede assistencial; a dinâmica dos serviços; e o perfil e formação dos agentes que são responsáveis por executar esta articulação. Porém, os profissionais atuantes no NASF-AB identificam a importância desta articulação considerando que as situações de saúde manejadas no contexto da ABS relacionam-se com questões sociais que necessitam de outros atores e setores envolvidos para que possa ser produzido o cuidado ao usuário.

As dificuldades encontradas para a utilização desta diretriz na ABS poderiam ser manejadas ao utilizar outras ferramentas preconizadas para o processo de trabalho das equipes do NASF-AB, como por exemplo, o ecomapa para facilitar a identificação de possíveis equipamentos da rede que possam servir de apoio para o cuidado do usuário que está sendo acompanhado, sendo pactuado com as equipes destes serviços ações que poderiam ser realizadas, utilizando como estratégia o projeto terapêutico singular e/ou o projeto de saúde no território.

\section{CONCLUSÕES}

O objeto de ação do contexto da ABS agrega elementos biológicos, psíquicos e sociais. Por este motivo, para entender e atuar sobre as situações existentes no território é necessário que os profissionais utilizem todas as ferramentas disponíveis e que articulem com vários setores como: educação e assistência social.

Também é necessário refletir sobre possíveis estratégias para lidar com as barreiras e dificuldades, que são encontradas no contexto da ABS, de modo a possibilitar a operacionalização do uso de ferramentas assistenciais e tecnológicas.

Apesar das barreiras e dificuldades encontradas, as características e dinâmica do processo de trabalho no contexto da $\mathrm{ABS}$ como a integração das equipes de $\mathrm{SF}$ e do NASF- AB se configuram como um facilitador para que o cuidado prestado ao usuário neste nível de atenção seja integral e resolutivo.

Agradecimentos: Aos participantes da pesquisa, ao Departamento de Terapia Ocupacional da UFPR e ao Programa de Residência Multiprofissional em Saúde da Família da UFPR.

Agência de fomento: Ministério da Saúde.

Participação dos autores: Derivan Brito da Silva, autor principal, responsável pelo delineamento do projeto, análise e interpretação dos dados, redação e revisão do artigo. Priscila Moreira, coautora, responsável pelo delineamento do tema, coleta, análise e interpretação dos dados e redação do artigo. Lilian Cristina dos Santos, colaboradora, responsável pela revisão do artigo. Maria José Gugelmin de Camargo, colaboradora, responsável pela revisão do artigo e contribuições teóricas. Denny Rafael Perusso dos Reis, colaborador, responsável pela revisão do artigo e contribuições práticas.

\section{REFERÊNCIAS}

1. Brasil. Ministério da Saúde. Portaria GM/MS n. ${ }^{\circ} 2.436$, de 21 de setembro de 2017. Aprova a Política Nacional de Atenção Básica, estabelecendo a revisão de diretrizes para a organização da Atenção Básica, no âmbito do Sistema Único de Saúde (SUS). Diário Oficial da União, Brasília; 2017. Disponível em: http://www.imprensanacional.gov.br/materia/-/ asset publisher/Kujrw0TZC2Mb/content/id/19308123/do1-2017-0922-portaria-n-2-436- de-21-de-setembro-de-2017-19308031.

2. Faria HP, Werneck MAF, Santos MA, Teixeira, PF. Processo de trabalho em saúde. 2a ed. Belo Horizonte: Nescon/UFMG; 2009. Disponível em: https://www.nescon.medicina.ufmg.br/ biblioteca/imagem/1790.pdf.

3. Brasil. Ministério da Saúde. Secretaria de Atenção à Saúde. Departamento de Atenção Básica. Diretrizes do NASF - Núcleo de Apoio a Saúde da Família. Brasília: Ministério da Saúde;
2009. (Série B. Textos Básicos da Saúde/ Cadernos de Atenção Básica; n. 27). Disponível em: http://bvsms.saude.gov.br/bvs/ publicacoes/caderno_atencao_basica_diretrizes_nasf.pdf.

4. Brasil. Ministério da Saúde. Secretaria de Atenção à Saúde. Departamento de Atenção Básica. Cadernos de Atenção Básica n. ${ }^{\circ}$ 39: Núcleo de Apoio à Saúde da Família. Brasília: Ministério da Saúde; 2014. Disponível em: http://bvsms. saude.gov.br/bvs/publicacoes/nucleo_apoio_saude_familia cab39.pdf.

5. Paim JS. Modelos de atenção à saúde no Brasil. In: Giovanella L, et al. Políticas e sistema de saúde no Brasil. Rio de Janeiro: Fiocruz; 2008. p.547-73.

6. Gil AC. Métodos e técnicas de pesquisa social. 5a ed. São Paulo: Atlas; 1999. 
7. Dyniewicz AM. Metodologia da pesquisa em saúde para iniciantes. 3a ed. São Caetano do Sul, SP: Difusão Editora; 2014.

8. Marconi MA, Lakatos EM. Metodologia do trabalho científico: procedimentos básicos, pesquisa bibliográfica, projeto e relatório, publicações e trabalhos científicos. 6a ed. São Paulo: Atlas; 2001.
9. Bourdieu P. O poder simbólico. 6a ed. Rio de Janeiro: Bertrand Brasil; 2003. p.51-8.

10. Minayo MCS. O desafio do conhecimento: pesquisa qualitativa em saúde. 8a ed. São Paulo: Hucitec; 2004.

11. Minayo MCS. O desafio do conhecimento: pesquisa qualitativa em saúde. 14a ed. São Paulo: Hucitec; 2014. 